\title{
Diagnosis challenge face to chronic spinal cord infarction
}

\begin{abstract}
Infarction of the spinal cord is no common. The MR Imaging helps to make diagnosis. We describe a clinical case of chronic spinal cord infarction. A young man presents a complaining of progressive right cervical and lumbar neuralgia with difficulty for walking. The diagnosis roamed for a long time but the mode of installation in "stair step" pleads for a chronic spinal cord infarction. We must keep in mind the possible discussion of a chronic spinal cord infarction in front of clinical signs of progressive myelo-radiculopathies and typical images of spinal cord's MRI.
\end{abstract}

Keywords: diagnosis, chronic infarction, spinal cord

\author{
Volume 8 Issue 4 - 2018 \\ Prisca-Rolande Bassolé,,,2 Mbagnick \\ Bakhoum,' Maoully Fall, 1,3 Moustapha \\ Ndiaye,' Massar Diagne,' Amadou Gallo \\ Diop,' Mouhamadou Mansour Ndiaye ${ }^{1,2}$ \\ 'Neurology Department of Fann Teaching Hospital, Senegal \\ ${ }^{2}$ Neurology department of El Hadj Ibrahima Niasse University, \\ Senegal \\ ${ }^{3}$ Neurology Department of Pikine Hospital, Senegal
}

Correspondence: Prisca-Rolande Bassolé, Neurology Department of Fann Teaching Hospital, Dakar, Senegal,Tel +22 I 77 I80 7 I 31, Email rolandebassole@gmail.com

Received: March 14, 2018 | Published: August 13,2018

\section{Introduction}

Spinal cord injury occurs when for any reasons; there is a damage of the spinal cord. This is a therapeutic emergency because of the fear of a roots or spinal cord compression engaging the functional or vital prognosis. Spinal cord injury constitutes a veritable challenge for positive and etiological diagnosis because there are reversible causes. Infarction of the spinal cord is no common and may have different clinical manifestations. ${ }^{1}$ The MR Imaging of spinal cord helps to make diagnosis. ${ }^{1,2}$ In this clinical case, we describe an atypical clinical presentation of cervical spinal cord infarction.

\section{Clinical case}

A 45 year-old man presents, in 2015, to his orthopedic surgeon, a complaining of a quickly progressive installation of cervical pain radiating in the three first fingers of right upper limb and pains in the right lower limb with difficulty for walking with this limb since more than four years. There is no concept of trauma or particular personal history.

The first practitioner thought to peripheral nerve disorders and asked a lumbar scan and ENMG. The lumbar Scan performed showed degenerative osteoarthritis of the hinge lumbar without disco radicular conflict. ENMG was normal but Evoked Somesthesic Potential (PES) showed right S1 radiculopathy. After one month of unsuccessful treatments, the first cervical MRI is performed and showed one T2-weighted sagittal midline image with elevated signal within the cord at the C3-C4 level over a length of $30 \mathrm{~mm}$ with the loss of cord volume. The $\mathrm{T} 2$-weighted axial image at the level of $\mathrm{C} 3$ demonstrates bilaterally symmetric well-circumscribed circular shaped foci with increased signal intensity. The appearance approximates the shape of a snake's eyes (Figures 1-3).

He comes to neurology consultation in october 2017, given the persistence of pains and deficit despite the analgesic and antiinflammatory treatments associated with physiotherapy. Clinical neurological examination shows a walking by mowing with the right lower limb. Non-proportional right hemiparesis more important on the lower limb. Bilateral hyperreflexia with bilateral Babinski and
Hoffman signs, bilateral amyotrophy of the proximal muscles, tactile hypoesthesia in the right hemibody, algesic hypoesthesia in the left hemibody, no vibration and position sense perception, a sensory ataxia and no cranial nerves disorders. The new spinal cord MRI performed in 2017 shows the same images in cervical level with more atrophy (Figures 1-3). Brain MRI is normal. The blood cell count shows WBC:4, 2g/L, Hemoglobin:13,9g/100ml, MCV:92 $\mu 3$, Platelet:264g/1 ; Blood erythrocyte sedimentation rate $(1 \mathrm{H}): 4 \mathrm{~mm}$; C-Reactive protein:0,4mg/l; Negatif human immuno-deficiency virus test.

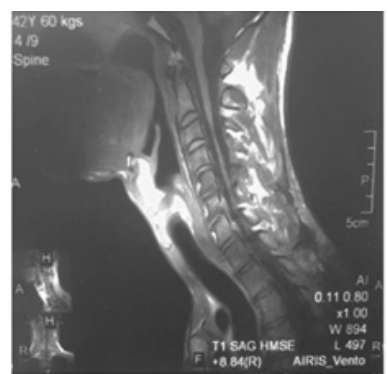

A)

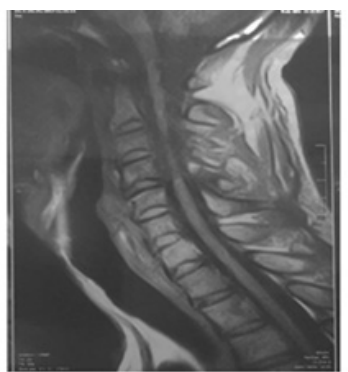

B)
Figure I TI-weighted sagittal image with loss of cervical level C3-C4 cord volume - A) 2015 spinal cord MRI -B) 2017 spinal cord MRI.
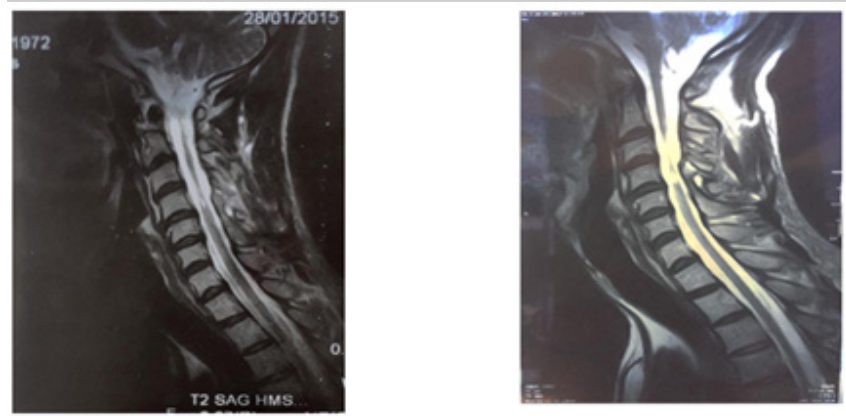

Figure 2 T2-weighted sagittal midline image demonstrates elevated signal within the cord at the C3-C4 level over a length of $30 \mathrm{~mm}$ with the loss of cord volume. - A) 2015 spinal cord MRI -B) 2017 spinal cord MRI. 

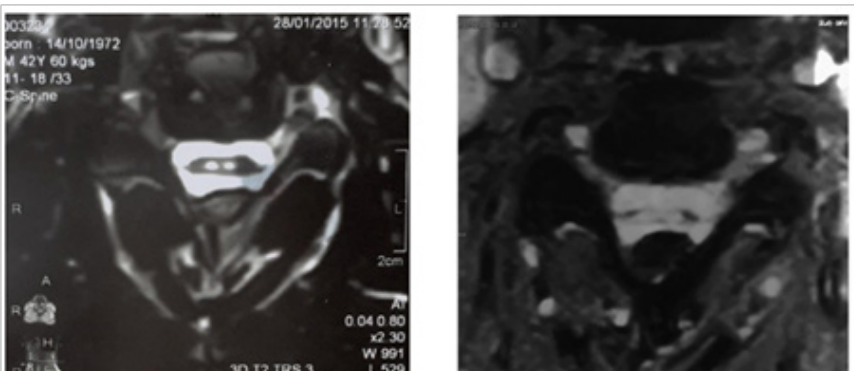

Figure 3 T2-weighted axial image at the level of $\mathrm{C} 3$ demonstrates bilaterally symmetric well-circumscribed circular shaped foci of increased signal intensity. The appearance approximates the shape of a snake's eyes.

A) 2015 spinal cord MRI -B) 2017 spinal cord MRI.

\section{Discussion}

Spinal Cord Infarction (SCI) is relatively rare but, however, still represents one of the most common causes of acute non compressive myelopathies. ${ }^{3}$ Clinical presentation depends mainly on the location and extent of the infarction. As with cerebral infarction, the onset of spinal cord infarction is typically abrupt. The neurological presentation of spinal cord infarction is largely defined by the vascular territory involved.

The root-type pains of this patient made the diagnosis roam for a long time but the mode of installation in "stair step", consisting of a progressive extension of the deficit associated with pains pleads for a chronic spinal cord infarction. Indeed, the clinical expression of chronic spinal cord infarction may correspond to progressive ischemic myelopathy with an array of myelo-radiculopathy whose initial signs are root-type pains and walking disturbances. ${ }^{4}$ The neurological presentation is in favor of a cervical injury of anterior spinal artery. According to the literature, the most common clinical presentation of a spinal cord infarction is anterior spinal artery syndrome..$^{1,5}$ The onset is usually abrupt, often marked by acute pain preceding the onset of the neurological deficit, sometimes with a progressive worsening. ${ }^{5}$ Anterior spinal artery infarct typically presents as a bilateral loss of motor function and pain/temperature sensation, with relative sparing of proprioception and vibratory senses below the level of the lesion. ${ }^{1}$ However, an infarction in the territory of the anterior spinal artery can lead to arthrokinetic and vibratory disorders because this artery vascularizes the deep part of the posterior cords. ${ }^{5}$

MRI evaluation is essential in the diagnosis of SCI. Abnormalities on T2-weighted sequences are seen in more than $90 \%$ of SCI cases. ${ }^{6}$ Nevertheless, these changes are not specific, and it is often difficult to distinguish SCI from other causes of acute non compressive myelopathies based on MRI alone. Sagittal MR images of anterior spinal artery infarction usually demonstrate an isolated pencil-like area of T2-hyperintensity involving the centromedullary region, often encompassing more than 2 vertebral segments. ${ }^{3,6}$ Because of the higher vulnerability of the gray matter to ischemia, axial T2sequences may show bilateral hyperintensities that are mostly confined to the anterior horn area, leading to the typical snake eyes or owl's eyes configuration. ${ }^{6,7}$ In the chronic stages, localized atrophy is possible, ${ }^{1}$ like in this case study, making it difficult to accurately locate hyperintensities in the anterior horn area in axial T2-sequences.

\section{Conclusion}

The observation which we described is relative to a chronic anterior spinal artery infarction with a progressive and insidious mode of installation, initially mimicking a radiculopathy.

The interest of this case lies in the wandering of the initial etiological diagnosis. We must keep in mind the possible discussion of a chronic spinal cord infarction in front of clinical signs of progressive myelo-radiculopathies and typical images of spinal cord's MRI.

\section{Acknowledgements}

None.

\section{Conflict of interest}

The author declares no conflict of interest.

\section{References}

1. Vargas MI, Gariani J, Sztajzel R, et al. Spinal cord ischemia: practical imaging tips, pearls, and pitfalls. Am $J$ of Neuroradiol. 2015;36(5):825830 .

2. Nogueira RG, Ferreira R, Grant PE, et al. Restricted diffusion in spinal cord infarction demonstrated by magnetic resonance line scan diffusion imaging. Stroke. 2012;43(2):532-535.

3. De Seze J, Stojkovic T, Breteau G, et al. Acute myelopathies: Clinical, laboratory and outcome profiles in 79 cases. Brain. 2001;124(pt 8):15091521

4. Masson C, Leys d, Meder JF, et al. Ischémie médullaire. J Neuroradiol. 2004;31(1):35-46.

5. Leys D, Cordonnier C, Masson C. Pruvo medullaire. EMC-Neurologie. $2005 ; 2: 163-174$

6. Infarctus JP, Fortuna A, Ferrante L, et al. Spinal cord ischemia diagnosed by MRI. Case report and review of the literature. J Neuro-radiol. 1995;22(2):115-122.

7. Weidauer $\mathrm{S}$, Nichtweiss M, Lanfermann $\mathrm{H}$, et al. Spinal cord infarction: MR imaging and clinical features in 16 cases. Neuroradiol. 2002;44(10):851857. 